Rabaska

Revue d'ethnologie de l'Amérique française

\title{
Marius Barbeau et l'authenticité de la tradition en art populaire
}

\section{Jean-François Blanchette}

Volume 13, 2015

Présence de Marius Barbeau : l'invention du terrain en Amérique

française. Autour d'un legs centenaire (1914-2014)

URI : https://id.erudit.org/iderudit/1033757ar

DOI : https://doi.org/10.7202/1033757ar

Aller au sommaire du numéro

Éditeur(s)

Société québécoise d'ethnologie

ISSN

1703-7433 (imprimé)

1916-7350 (numérique)

Découvrir la revue

Citer cet article

Blanchette, J.-F. (2015). Marius Barbeau et l'authenticité de la tradition en art populaire. Rabaska, 13, 141-151. https://doi.org/10.7202/1033757ar d'utilisation que vous pouvez consulter en ligne.

https://apropos.erudit.org/fr/usagers/politique-dutilisation/ 


\title{
Marius Barbeau et l'authenticité de la tradition en art populaire ${ }^{1}$
}

\author{
Jean-François Blanchette
}

Chercheur associé, Musée canadien de l'histoire

\section{Introduction}

La recherche des traditions en art populaire et de leurs origines à l'époque même où la modernité les menace de disparition amène Marius Barbeau à mettre ces traditions en valeur et à favoriser leur persistance. Ses actions trouvent leur écho à l'époque de la crise économique où justement certains prônent le retour à la terre et aux activités domestiques qui permettraient de subvenir aux besoins de la famille. Par ailleurs, l'approche de l'anthropologue et folkloriste est confrontée à la vision de certains qui croient, comme Jean-Marie Gauvreau, qu'il faille jumeler certaines de ces traditions avec des éléments de modernité afin de créer des produits artisanaux pour le marché.

Jean-Marie Gauvreau est au centre d'un mouvement de renouveau artisanal au moment où s'effrite la production traditionnelle. Lui-même intéressé par les découvertes de Barbeau sur le terrain, il finira cependant par diverger sur l'action à entreprendre. Gauvreau croit qu'il faut donner un coup de barre, fournir des directives sur ce qu'il faut produire et comment le produire tout en étant à l'écoute de la clientèle. Ce mouvement aura comme effet de favoriser une production artisanale commerciale correspondant au nouvel esthétisme que favorise Gauvreau. Ce dernier représente non seulement une école officielle mais aussi une école de pensée qui prône l'influence de la culture élitiste française de l'époque. De plus, il a l'écoute du gouvernement et peut aider à délier les bourses pour favoriser la petite entreprise et l'artisanat. Ce qui guide Gauvreau, c'est l'enseignement reçu des maîtres qui l'ont formé à l'école Boulle, l'une des grandes écoles d'ébé-

1. Je tiens à remercier Benoît Thériault du Musée canadien de l'histoire pour les nombreuses références au Fonds Marius Barbeau qu'il m'a données lors de cette recherche ainsi que Nicole Bourgault pour ses communications concernant la correspondance entre Marius Barbeau et Médard Bourgault. 
nisterie de la France où on formait ébénistes, menuisiers en siège, tapissiers et sculpteurs sur bois. Une école qui avait fait sa renommée dans la copie d'œuvres anciennes, particulièrement du mobilier de style Louis XV. Gauvreau apprécie le savoir-faire issu de cette période. C'est cela qu'il est venu chercher en France et qu'il veut transposer au Québec. Il n'ignore pas l'importance des traditions du Canada français, bien au contraire. Il insiste pour qu'on documente les techniques anciennes et qu'on s'intéresse à nos vieilles artisanes.

À lire ce que dit Jean-Marie Gauvreau des traditions et du travail de Marius Barbeau, on croirait que les deux baignent dans le même paradigme. Gauvreau vante les tapis crochetés des grands-mères, aux dépens de ceux qu'on fabrique désormais à partir de modèles commerciaux :

Et pas un de ces tapis ne ressemblait à ceux de la voisine. Les fermières usaient d'ingéniosité pour en faire un à leur idée ! Et comment ne pas évoquer, à l'examen des anciens modèles de tapis crochetés, rassemblés par l'infatigable Marius Barbeau, combien nos vieilles grand-mères, d'il y a cinquante ans et plus, n'étaient jamais à bout de ressources. Même naïvement dessinés, tous les animaux de la ferme défilaient à tour de rôle en des paysages qui feraient envie à ceux qu'on qualifie de primitifs. Quand la nature ne les inspirait pas, dans un journal plié en quatre ou en huit épaisseurs, ciseaux en main, elles découpaient machinalement d'ingénieux motifs, plus ou moins figuratifs, lorsque les épaisseurs étaient dépliées. Et dire qu'au $20^{\circ}$ siècle, on revendique d'avoir découvert l'automatisme !?

Pourtant, avec le recul du temps et la nouvelle perspective que nous permet l'accès aux documents anciens, on se rend compte que les deux protagonistes ont des visions bien différentes.

\section{Le contexte du renouveau artisanal}

La crise économique qui survient en 1929 amène les politiciens, les hauts fonctionnaires, les dirigeants des grandes écoles et tous ceux qui sont engagés socialement, à chercher des moyens afin de donner de l'ouvrage à la population et sortir les gens de la grande misère. Plusieurs voient en l'artisanat une source de travail et de fierté pour la population avec la possibilité de le développer à l'intérieur de petites entreprises.

La tâche n'est pas facile car les traditions se sont effritées dramatiquement depuis le siècle précédent. Barbeau en témoigne quand il publie sa synthèse sur les tapis crochetés en 1947, où il mentionne que les tapis crochetés sont désormais tous de type touristique inspirés de calendriers

2. BAnQ-Montréal, Fonds Jean-Marie Gauvreau, MSS2/1/23 f), Jean-Marie Gauvreau, «L'Artisanat du Québec » (4 copie), Mémoire présenté à la Société royale du Canada, Halifax, 5-8 juin 1949 , p. 26-27. 
et catalogues commerciaux ${ }^{3}$. Les effets de la modernité sont évidents aussi pour Clarence Gagnon :

Au cours du dernier siècle, l'industrialisation a fait sa marque inévitable au pays. Le déclin du bon goût et l'arrivée massive de biens industrialisés a causé un tort irréparable à la production artisanale, au point que la disparition totale de l'art paysan sera bientôt un fait accompli. ${ }^{4}$

Pour sa part, Marius Barbeau est plutôt intéressé par l'identification des anciennes traditions canadiennes de source française et par la persistance des dites traditions. Dès $1927^{5}$ et $1928^{6}$, il participe activement à l'organisation de festivals avec le Canadien Pacifique afin de faire la promotion des arts domestiques à une clientèle étatsunienne qui visite le Québec. Ce faisant, il souhaite que le contact avec les touristes favorise la reconnaissance de ces traditions françaises originales implantées puis développées dans la vallée $\mathrm{du}$ Saint-Laurent depuis plus de trois siècles. Mais il vise aussi à faire reconnaître ces traditions ancestrales par l'élite culturelle canadienne-française qui n'a d'œil que pour les modernités venues de France ${ }^{7}$. Barbeau croit aussi au développement commercial des arts, pourvu qu'on crée de la qualité et qu'on ne reproduise pas les œuvres à outrance ${ }^{8}$.

Tandis que Barbeau visite Charlevoix pour y identifier les traditions, Jean-Marie Gauvreau et Paul Gouin, qui s’y rendent en 1933, comptent plutôt y trouver des artisans qui pourraient être encouragés à développer leurs talents à des travaux économiquement rentables. Cette mission de Gauvreau et Gouin dans la région de La Malbaie semble découler d'une critique que Gouin avait faite l'année précédente sur une exposition de Gauvreau, qui est alors professeur d'ébénisterie et chef de la section du meuble à l'École technique de Montréal ${ }^{9}$ où l'inspiration ne provenait pas des meubles québécois anciens mais plutôt de meubles français. Le politicien Paul Gouin avait d'ailleurs comme base de sa plateforme socioéconomique le déve-

3. Marius Barbeau, «The Origin of the Hooked Rug », Antiques, vol. 52, n 2, août 1947, p. 111 (article soumis en 1944).

4. Clarence Gagnon, «For a Revival of Canadian Folk Art », Manuscrit, 1939. BanQ-Montréal, Fonds Jean-Marie Gauvreau, MSS2/3/7, p. 5-6. Traduction personnelle.

5. Festival de la chanson, des danses et des métiers du terroir, Québec, Château Frontenac, 20-22 mai 1927. Musée canadien de l'histoire, Fonds Marius Barbeau, CDA-2004-2-16, 17,18.

6. Festival de la chanson et des métiers du terroir, Château Frontenac de Québec, 24-28 mai 1928. Musée canadien de l'histoire, Fonds Marius Barbeau, fonds électronique, CDA-2004-2-22.

7. On lira à ce sujet Richard Handler, Nationalism and the Politics of Culture in Quebec, Madison, The University of Wisconsin Press, 1988, 217 p., et Daniel Guilbert, «La légende des "Veillées du bon vieux temps" ( $1^{\text {ere }}$ partie) », vendredi 30 mars 2012, (novembre 2010), http://www.mnemo.qc.ca/ spip/bulletin-mnemo/article/la-legende-des-veillees-du-bon-vieux-temps.

8. Marius Barbeau, «La Charité bien ordonnée... », La Patrie, 6 juin 1954, p. 50-51. Musée canadien de l'histoire, Fonds Marius Barbeau, B152.

9. C'est à ce titre qu'il donne une conférence le 2 juillet 1932. Bibliothèque et Archives Canada, Fonds Paul Gouin, MG27-IIID1, vol. 22, dossier 41. 
loppement de la petite industrie à partir des traditions québécoises. Gouin amène ainsi Gauvreau à découvrir les traditions québécoises qui lui seront utiles à l'enrichissement de sa pensée et à l'établissement de son programme scolaire sur des bases sociopolitiques acceptables ${ }^{10}$.

\section{L'École du meuble}

Une École du meuble, école des « arts appliqués aux industries de l'ameublement », est fondée en 1935 par Jean-Marie Gauvreau qui en est le premier directeur. Cette école a comme mandat la formation professionnelle de jeunes artisans et artisanes de l'ébénisterie et des métiers artisanaux qui y sont rattachés comme la sculpture et la tapisserie ${ }^{11}$. Les activités de cette école sont observées de près par divers ministères intéressés par la petite industrie, l'artisanat, le commerce et le tourisme, car ils espèrent que cette école contribuera à la nouvelle dynamique économique que le gouvernement veut créer pour enrayer les effets néfastes de la crise économique qui sévit à ce moment-là. La formation d'une main-d'œuvre spécialisée contribuera, croit-on, au développement de petites entreprises. De plus, on espère raviver la créativité des artisans et artisanes dont on vante la débrouillardise et le savoir-faire observés par le passé. Ce n'est donc pas un hasard si, en 1937, lors de l'organisation des équipes qui vont participer à l'inventaire des ressources naturelles du Québec mis en place par le gouvernement de Maurice Duplessis et dont la responsabilité a été confiée à Esdras Minville, directeur des Hautes études commerciales, ce dernier demande à Gauvreau de diriger l'une des équipes de l'inventaire. Gauvreau convaincra Minville de le charger d'un inventaire spécialisé de l'artisanat l'année suivante. Gauvreau engagera ainsi Albert Tessier et Paul-Émile Borduas à collaborer avec lui à un début d'inventaire des ressources artisanales du Québec à l'été 1938. Et comme le Ministre de l'industrie et du commerce est convaincu de l'importance du tourisme dans le développement économique du Québec, on leur donne aussi le mandat d'observer et documenter la situation de cette industrie ${ }^{12}$.

$\mathrm{Au}$ cours de ces enquêtes de terrain dans les comtés visités le long du Saint-Laurent au nord-est de Québec et en Gaspésie, Gauvreau dit que ses enquêteurs et lui ne manquaient pas de donner des conseils et Tessier de

10. Voir Nathalie Hamel, "Notre maitre le passé, notre maitre l'avenir », Paul Gouin et la conservation de l'héritage culturel du Québec, Québec, Presses de l'Université Laval, 2008, 204 p.

11. La tapisserie dont il est question ici est l'art du recouvrement des sièges.

12. L'inventaire des ressources artisanales du Québec est discuté en profondeur dans Jean-François Blanchette, Du coq à l'âme, l'art populaire au Québec, Gatineau, Musée canadien de l'histoire et Ottawa, Presses de l'Université d'Ottawa, collection « Mercure, Études culturelles » 85, 2014, p. $72-88$. 
faire des démonstrations du savoir-faire grâce à ses films et à une collection de belles pièces présentées à un public évalué à plus de 7000 personnes. En effet, Albert Tessier donne des conférences et fait des démonstrations avec de bons ouvrages qu'il a apportés avec lui. Ces présentations permettaient de « mettre en valeur la noblesse du travail et la fierté que nous devons éprouver autant que la satisfaction dans le devoir accompli, leçons que nous ont laissées nos ancêtres. ${ }^{13}{ }^{\prime \prime}$

\section{À la suite de ces enquêtes, Gauvreau convainc le gouvernement de créer} des ateliers-écoles afin de former des artisans et artisanes à des métiers pour la création de produits qui vont répondre à la demande du marché touristique et des marchés extérieurs. Ainsi, des centaines d'artisans et d'artisanes vont être formés à des métiers rémunérateurs dans des ateliers-écoles comme ceux qui ont été subventionnés par le Service de l'aide à la jeunesse à SaintJean-Port-Joli pour la sculpture sur bois ${ }^{14}$, à Pointe-au-Pic pour les tapisseries ${ }^{15}$, à Saint-Joseph-de-Beauce ${ }^{16}$ et à Chicoutimi ${ }^{17}$ pour la céramique et à Saint-Barthélemy ${ }^{18}$ pour la fabrication du jouet. On reçoit aussi une for-

13. BANQ-Montréal, MSS2/Fonds Jean-Marie Gauvreau, Jean-Marie Gauvreau, «Rapport général sur l'artisanat, ou Mémoire sur l'artisanat dans la Province de Québec », Québec, Ministère des Affaires municipales, de l'industrie et du commerce, 26 avril 1939, p. 1-2.

14. Médard Bourgault sculptait déjà depuis les années 1920. Son frère Jean-Julien se joignit à lui en 1931, leur frère André peu après, puis un quatrième frère, Antonio, ainsi que leur neveu Léon Toussaint, sans compter leur jeune sœur Yvonne, et finalement Raymond, fils de Médard. Son frère Jean-Julien se joignit à lui en 1931, leur frère André peu après, puis un quatrième frère, Antonio, ainsi que leur neveu Léon Toussaint, sans compter leur jeune sœur Yvonne, et finalement Raymond, fils de Médard.. C'est en novembre 1940 qu'une école subventionnée par le gouvernement ouvrit ses portes. Médard et Jean-Julien en furent les professeurs, Jean-Marie-Gauvreau le directeur pédagogique. Voir Jean-Marie Gauvreau, Artisans du Québec, Trois-Rivières, Éditions du Bien Public, 1940, p. 93-140, et les répertoires numériques détaillés des Fonds Médard Bourgault et Jean-Julien Bourgault aux Archives de la Côte-du-sud.

15. Georges-Édouard Tremblay, appuyé par son épouse et avec l'aide de plusieurs artisanes, fabriquaient déjà des tapis crochetés depuis au moins 1931. Il transforma la technique du tapis crocheté traditionnel pour en faire des tapisseries et, en raison de la demande, de grands et magnifiques tapis de plancher. L'atelier fit place à l'école en 1942. Voir Richard Dubé et François Tremblay, Peindre un pays. Charlevoix et ses peintres populaires, Montréal, Broquet, 1989, p. 128-148.

16. Cette école fut inaugurée en novembre 1940 en partenariat avec le Syndicat des céramistes paysans de la Beauce. Elle donnait une nouvelle vie au centre artisanal de Saint-Georges de Beauce fondé en 1933 et dirigé par Oscar Bériau de l'École des arts domestiques de Québec. Voir Daniel Cogné, Richard Dubé et Paul Trépanier, Céramique de Beauce, Québec, GiD, 2004, p. 13-38 ; BanQ-Montréal, MSS2/Fonds Jean-Marie Gauvreau : Jean-Marie Gauvreau, « Le Problème de l'artisanat de la Province de Québec. Étude présentée au Ministère de l'Industrie et du Commerce de Québec », manuscrit inédit, 1942, p. 116-120.

17. L'école de céramique de Chicoutimi, Poterie saguenayenne, est moins connue que les précédentes. Un atelier de céramique avait vu le jour grâce au soutien financier du marchand local Jos Gagnon, puis a reçu une subvention de l'Aide à la jeunesse en 1940 (cf. Gauvreau, op. cit., 1942, p. 121-123).

18. L'école de fabrication de jouet de Saint-Barthélemy est peu connue. Elle a vu le jour grâce à l'initiative du frère J.W. Pomerleau, c.s.v., directeur de l'École moyenne d'agriculture de SaintBarthélemy (Lanaudière) et grâce à une subvention de l'Aide à la jeunesse en 1940-1941 (cf. Gauvreau, op. cit., 1942, p. 80-84). 


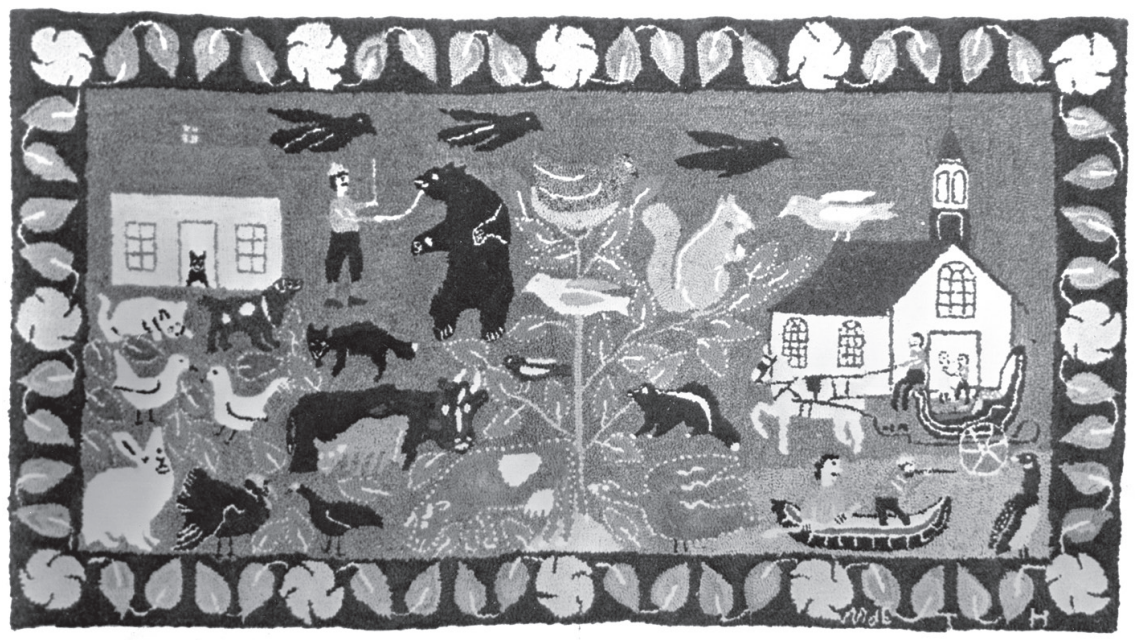

Tapis au crochet fabriqué par madame Marie-Louise (Tremblay) Hovington de Tadoussac, Québec. Scène villageoise (à droite : la chapelle de Tadoussac). 1938. Source : Musée canadien de l'histoire, n 82264.

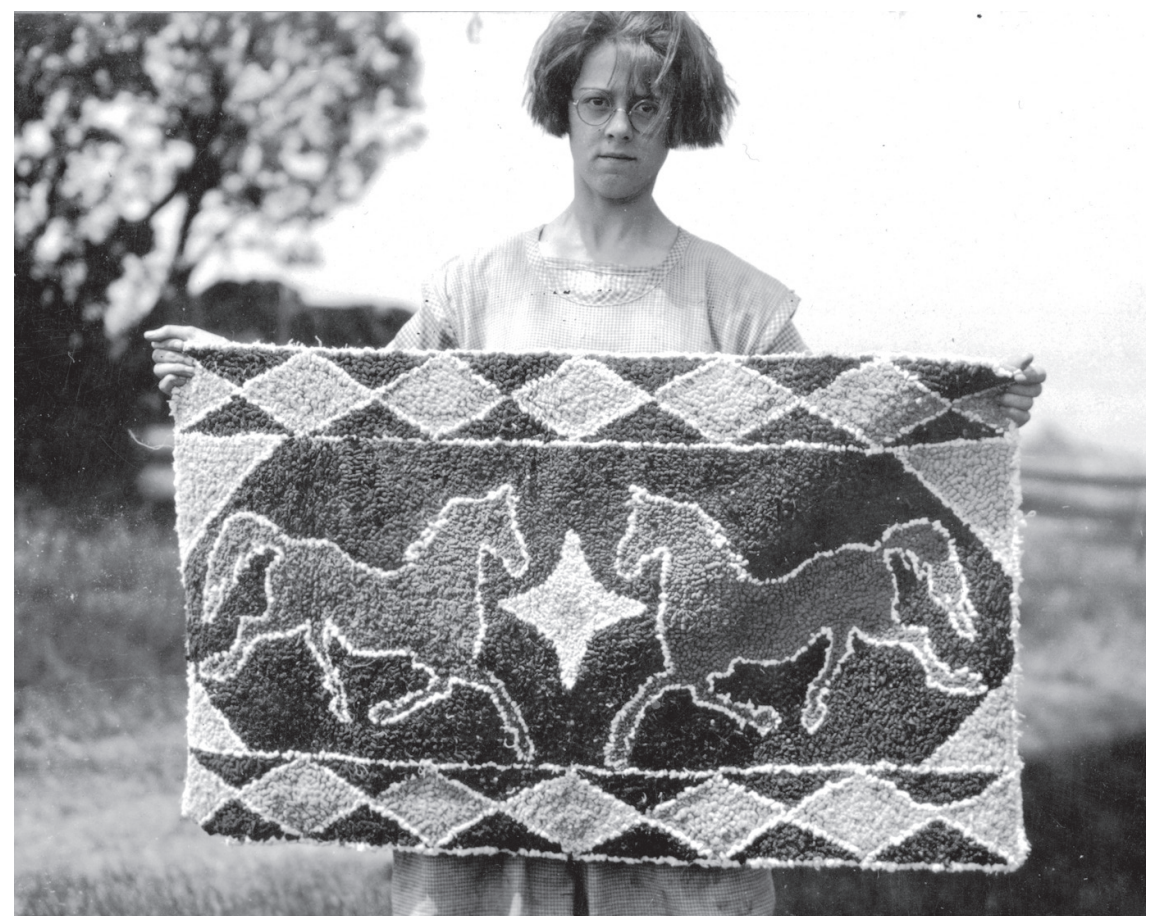

Tapis au crochet fait (récemment) par madame René (Adéla) Sanschagrin, Saint-François, Île d'Orléans, Québec, 1925.

Source : Musée canadien de l'histoire, nº 65682. 
mation par les divers organismes ou écoles qui fourmillent au Québec lors de cette période. On valorise le " savoir-faire du beau », dans le meuble, la sculpture, la peinture, l'outillage, les jouets, les textiles, etc.

\section{Tradition - innovation}

C'est au cours des années 1930 que les orientations de Barbeau et Gauvreau commencent à se distinguer. Gauvreau est élogieux sur le travail de Barbeau et les œuvres qu'il collectionne parce que celles-ci démontrent le savoirfaire des anciens Canadiens. Il est par ailleurs très critique de l'orientation que ces traditions ont prise depuis quelques décennies. Il croit qu'il faut moderniser les techniques et modifier l'esthétique de la production artisanale. Il s'oppose ainsi à Barbeau dont l'approche scientifique favorise l'observation des traditions, leur documentation et leur diffusion afin d'en apprécier les qualités intrinsèques.

En 1938, lors de l'organisation des inventaires spécialisés en artisanat, Jean-Marie Gauvreau indique à Esdras Minville dans une lettre datée du 25 mai 1938 qu'il ne désire pas inviter Marius Barbeau à participer à ces enquêtes « pour des raisons qu'il lui indiquera en privé ${ }^{19}$ ». On a interprété que c'était parce que Barbeau était à ramasser des objets pour créer un musée pour le gouvernement fédéral et que sa présence parmi les enquêteurs nuirait à leur propre mission ${ }^{20}$. Il est vrai que Gauvreau désirait constituer une collection et que Marius Barbeau collectionnait les objets anciens pour le Musée national du Canada. Mais ce dernier offre régulièrement des collections à diverses institutions québécoises dont le Musée du Québec ${ }^{21}$, et l'École du meuble. Par exemple, en 1941 il expédie à Jean-Marie Gauvreau un total de 33 pièces dont 22 tapis crochetés que ce dernier achète pour l'École du meuble ${ }^{22}$. Barbeau, en effet, ne vise qu'à sauver ces témoins des traditions anciennes et il est heureux de collaborer avec Gauvreau et les autres qui participent à ce mouvement de sauvetage. Mais il semble bien que Gauvreau craint que l'objectif que lui-même s'est donné d'influencer la stylistique et la technique des œuvres produites ne vienne à contrecourant des intérêts scientifiques et de la vision même de Barbeau.

19. Copie carbone de lettre de Jean-Marie Gauvreau à Esdras Minville, conseiller technique, Ministère du Commerce et de l'industrie, Montréal, 25 mai 1938. Musée des Beaux-arts de Montréal, Fonds Paul-Émile Borduas, dossier 237.8.

20. André-G. Bourassa, Jean Fisette et Gilles Lapointe, Paul-Émile Borduas, Écrits I, Montréal, Presses de l’Université de Montréal, 1987, p. 65.

21. Dans ses mémoires, Marius Barbeau indique qu'il a collectionné des pièces d'artisanat anciennes pour le Musée de la province dès 1930. Voir Musée canadien de l'histoire, Fonds Carmen Roy, Acq. 2000-F0003, dossier « Les Mémoires de Marius Barbeau », Boîte 624 f.10, p. 209.

22. Ces pièces ont pour la plupart été acquises par Barbeau en 1937. Elles datent du siècle précédent. BANQ-Montréal, MSS2/3/1 et Musée canadien de l'histoire, Fonds Marius Barbeau, boîte B366, dossier « Artefacts 1937 ». 
Gauvreau n'apprécie guère en effet l'esthétique populaire qu'on retrouve dans les campagnes du Québec. Il critique ouvertement le style des tapis crochetés parce que, à son avis, les sujets traités manquent de perspective et qu'on utilise des couleurs criardes. Il propose que les artisanes ne créent pas elles-mêmes les modèles de leurs tapis, mais s'en remettent aux professeurs et spécialistes de l'École des beaux-arts et l'École des arts appliqués : «Il faut laisser aux spécialistes le soin d'élaborer des modèles, faire confiance à ceux qui ont des connaissances artistiques, qui maîtrisent à fond les lois de la composition décorative. ${ }^{23}$ » Il n'encourage pas non plus les peintres populaires comme Georges-Édouard Tremblay qui, à son sens, n'a du talent que pour les tapis crochetés :

Celui-ci [Georges-Édouard Tremblay] a des dons artistiques intéressants. Il peint à ses heures et a déjà vendu plusieurs de ses œuvres à l'occasion d'expositions locales. S'il veut bien accepter notre conseil, qu'il emploie ses moyens d'expression au service d'une spécialité artisanale où il excelle. ${ }^{24}$

Du coté de la sculpture, il décourage les artistes à peindre leurs œuvres, car cela leur donne l'allure du plâtre, qui a d'ailleurs envahi les marchés depuis les débuts de l'industrialisation et l'importation de produits faits en série. Il essaie de convaincre Yvonne Bolduc de ne plus peindre ses sculptures. Cette dernière lui réplique que justement ces dernières se vendent mieux et qu'il faut bien vivre ! ${ }^{25}$ D'ailleurs Médard Bourgault aussi peignait ses œuvres, comme en font foi les critiques de Gauvreau à son égard ${ }^{26}$ ainsi que les photos des œuvres qu'il expose à l'entrée de son atelier en 1934 et 1941-1942. L'aversion que Gauvreau a pour la polychromie des sculptures est conforme aux dictats de l'esthétisme classique du temps ${ }^{27}$.

Barbeau s'oppose à Gauvreau lorsque ce dernier vise à orienter l'esthétique de l'école de sculpture sur bois de Saint-Jean-Port-Joli que vient de subventionner le gouvernement et où enseignent Médard et Jean-Julien Bourgault. Il écrit à Médard pour s'en plaindre, car il voyait Médard comme l'héritier du grand sculpteur Louis Quévillon (1749-1823) qui avait enseigné la sculpture à l'arrière-grand-père de Médard, Amable Charron

23. Jean-Marie Gauvreau, op.cit., 1942, p. 86 ; BANQ-Montréal, MSS2/2/3, Jean-Marie Gauvreau, « Rapport général sur l'artisanat » à l'honorable Ministre des Affaires municipales, de l'industrie et du commerce, 26 avril 1939, Ms., p. 19.

24. Ibid., p. 89.

25. BanQ-Montréal, Fonds Jean-Marie Gauvreau, MSS2/6/5, Rapports d'enquêtes, comté de Charlevoix, Arts domestiques, 1940, p. 68.

26. Gauvreau, op. cit., 1942, p. 116.

27. Cette approche semble avoir eu raison de la production en sculpture populaire par la suite, car la majorité des sculptures animalières répertoriées au Québec entre 1977 et 1979 sont laissées au bois naturel. Voir Jean Simard, Bernard Genest, Francine Labonté et René Bouchard, Pour passer le temps : artistes populaires du Québec, Québec, Gouvernement du Québec, Ministère des Affaires culturelles, «Les Cahiers du patrimoine»17, 1985, $186 \mathrm{p}$. 
$(1785-1844)^{28}$. Barbeau aurait préféré que Médard continue à sculpter comme auparavant, dans le style des anciens sculpteurs de la NouvelleFrance. Il est déçu de constater que la nouvelle école de Saint-Jean-Port-Joli développe un autre style, plus approprié à la demande contemporaine et à celle des touristes, encouragée en cela par les professeurs de l'École du meuble et les subventions qui lui sont versées pour la formation d'artisans et sculpteurs $^{29}$. Barbeau ne lésine pas sur les conseils qu'il donne à Médard et il ose même comparer les travaux de ses frères qu'il n'apprécie guère. Médard Bourgault réagit favorablement à cette mise en garde de Barbeau, car il réalise que l'école sert à former des ouvriers autant que des artistes. Si bien qu'il viendra à afficher ouvertement sa dissidence d'avec la production commerciale qui sortira parfois de Saint-Jean-Port-Joli. Sa carte de visite, autour des années 1950, fait toujours la promotion de la sculpture sur bois d'art religieux, qui est devenue sa spécialité depuis 1935, mais il y inscrit au bas : «Ne pas confondre avec les Bourgault qui font le bibelot ${ }^{30}$ ».

L'opposition entre les approches de Marius Barbeau et de Jean-Marie Gauvreau est confirmée dans une lettre datée du 8 mai 1961, que Jean Palardy écrit à Marius Barbeau. Il lui envoie copie de son manuscrit sur les meubles du Canada français pour commentaires. Il écrit :

S'il vous vient à l'esprit, en lisant le manuscrit, de faire des remarques, ne vous gênez pas, je les apprécierai beaucoup, même si certaines de mes opinions ne s'accordent pas avec les vôtres.

Croyez-vous que je suis trop dur envers certaines personnes, dans ma conclusion? Je ne puis m'empêcher de viser deux personnes, Jean-Marie Gauvreau et Paul Gouin que je ne nomme point [dans cette conclusion]. Ces deux seuls sont responsables du faux renouveau artisanal chez nous.

Si vous pensez que je ne devrais pas les viser, dites-le[-]moi.

Mille mercis Marius, et mes meilleurs souhaits.

Sincèrement, Jean Palardy ${ }^{31}$

Dans la conclusion qu'il publie, Palardy inclut une section qui s'intitule «Tradition et esthétisme ». Il y traite de la perte des traditions françaises au Canada français après les années 1820, en raison de l'influence croissante des styles importés par les artisans anglais et écossais après la Conquête et

28. Jean-Marie Gauvreau, " Médard Bourgault et l'École de sculpture sur bois de Saint-Jean-Port-Joli », Technique, ${ }^{\circ}$ 15, Montréal, 1940, p. 87-98.

29. La subvention reçue du Programme d'aide à la jeunesse sert à défrayer les salaires des professeurs, la pension et la rémunération des étudiants. La production appartient par ailleurs à l'école qui la vend.

30. Archives de la Côte-du-sud, Fonds Médard Bourgault, F050/C.

31. BANQ-Montréal, Fonds Marius Barbeau, P51, Bobine 5084, négatif 1772. 
les loyalistes après la guerre de l'Indépendance américaine. Il écrit :

Nous avons perdu le fil de nombreuses traditions qui nous auraient permis d'évoluer normalement vers des réalisations originales et fortes. Cette exubérance et cette fantaisie dont nous étions si friands auraient pu se perpétuer, s'épanouir et orienter aujourd'hui notre production artisanale vers un style canadien authentique. ${ }^{32}$

Palardy critique le fait qu'on s'est peu intéressé à la promotion de ces traditions d'origine française. Il a fallu attendre l'entre-deux-guerres pour qu'un groupe de promoteurs tente un renouveau artisanal que Palardy critique en ces mots :

Et vers les années 1935, des conférenciers canadiens[-] français prêchèrent un retour à la petite industrie, aux métiers artisanaux en voie de perdition; et des personnes bien intentionnées, croyant sincèrement se rattacher aux traditions du passé, lancèrent dans tout le Canada français un mouvement de renouveau artisanal. Ce mouvement, malheureusement, démarra sans directives sérieuses et sans que l'on eût, au préalable, fait un inventaire de notre patrimoine dans ce domaine. Il en résulta des créations de mauvais goût empruntées aux sources les plus disparates. Ce renouveau artisanal s'avilit davantage quand on s'est mis à imiter sans discernement les nouveautés qui nous envahirent depuis la guerre. ${ }^{33}$

La critique de Palardy vise entre autres le manque de connaissances préalables pour donner une nouvelle orientation à l'artisanat : l'absence d'inventaire. Il y eut en effet quelques inventaires de l'artisanat entre 1937 et 1942 réalisés par Gauvreau et ses collaborateurs, mais les rapports de ces inventaires, qui ne furent que partiels, ne furent jamais diffusés dans le public. On les avait miméographiés seulement pour les divers services gouvernementaux qui en avaient besoin ${ }^{34}$. Un autre aspect de la critique de Palardy traite justement de l'orientation nouvelle donnée à l'artisanat. En effet, Gauvreau s'est assuré que tous les ateliers-écoles qui furent subventionnés par le gouvernement aient des professeurs venant de France afin d'enseigner les techniques et les styles officiels reconnus dans le vieux pays. Par conséquent, aux yeux de Palardy, cette formation ne tenait pas assez compte de la tradition qui s'était développée et implantée dans la vallée du Saint-Laurent.

32. Jean Palardy, Les Meubles anciens du Canada français, Montréal, Pierre Tisseyre, 1971 [Paris, Le Cercle du livre de France, 1963], p. 386.

33 Ibid., p. 387.

34. On en trouvera certains dans le Fonds Jean-Marie Gauvreau MSS2 à la BANQ-Montréal, les autres dans diverses bibliothèques. Voir Jean-François Blanchette, $D u$ coq à l'âme, l'art populaire au Québec, Gatineau, Musée canadien de l'histoire, et Ottawa, Presses de l'Université d'Ottawa, collection « Mercure, Études culturelles» 85, 2014, p. 72-88. 


\section{Conclusion}

Marius Barbeau consacra toute sa carrière à l'identification des traditions françaises qui s'étaient établies puis développées avec un caractère spécifique dans la vallée du Saint-Laurent. Ses publications, ses conférences et les collections qu'il acquit pour le Musée national du Canada et d'autres musées du Québec et du Canada visèrent la préservation de ce patrimoine matériel et immatériel des Canadiens français. Il en fit également la promotion autant qu'il a pu, essayant de convaincre les divers intervenants et le public de l'importance de ces traditions dans le développement des arts au pays. Il s'intéressa aussi à promouvoir ces traditions auprès des étrangers et des touristes. Mais il semble bien qu'il ne vit pas, au début du moins, l'effet pervers que les foires de promotion de l'artisanat traditionnel auprès des touristes auraient sur l'authenticité de cette production. Car la créativité populaire allait être fortement influencée par ces contacts avec les touristes et leur demande pour des produits typés qui n'avaient plus de rapport avec la vie traditionnelle locale. Qui plus est, certains de ceux sur qui il comptait pour apprécier ces traditions et les mettre en valeur, allaient orienter ces traditions vers la modernité et les éloigner ainsi à ses yeux de leur originalité. Jean-Marie Gauvreau avait été convaincu par Paul Gouin qu'il fallait tenir compte des traditions, mais tout au long de sa carrière Gauvreau allait privilégier l'influence de maitres venant de France afin de moderniser le savoirfaire, les techniques et l'esthétisme des arts domestiques pour en faire des arts décoratifs. Les protagonistes de la modernisation des arts domestiques allaient recevoir tout un appui lorsque la recommandation de Jean-Marie Gauvreau de créer un « organisme de promotion et de contrôle» de l'artisanat sera finalement adoptée par la création en 1945 de l'Office provincial de l'artisanat et de la petite industrie dont il sera le premier président. 